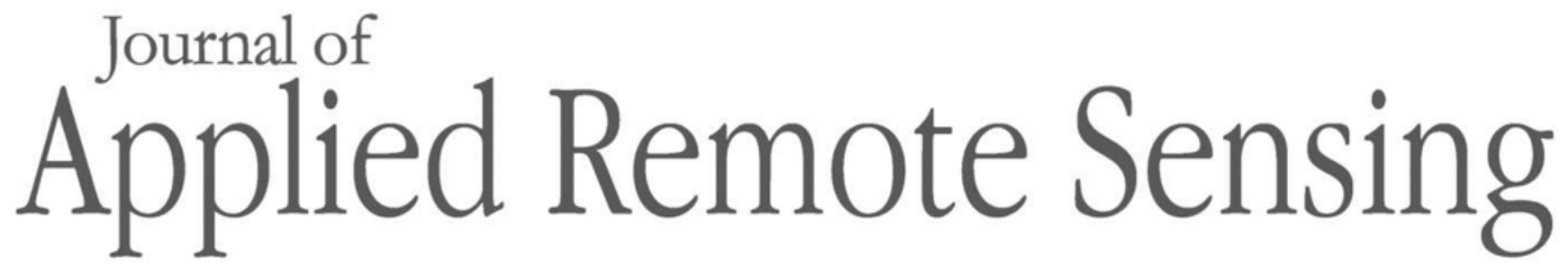

RemoteSensing.SPIEDigitalLibrary.org

\title{
Special Section Guest Editorial: Remote Sensing for Investigating the Coupled Biogeophysical and Biogeochemical Process of Harmful Algal Blooms
}

Ni-Bin Chang
Alan Weidemann

GPIE Coupled Biogeophysical and Biogeochemical Process of Harmful Algal Blooms," J. Appl. Coupled Biogeophysical and Biogeochemical Process of Harmful A
Remote Sens. 11(1), 012001 (2017), doi: 10.1117/1.JRS.11.012001. 


\title{
Special Section Guest Editorial: Remote Sensing for Investigating the Coupled Biogeophysical and Biogeochemical Process of Harmful Algal Blooms
}

\author{
Ni-Bin Chang a and Alan Weidemann ${ }^{\mathrm{b}}$ \\ ${ }^{a}$ University of Central Florida, Stormwater Management Academy, Orlando, \\ Florida 32816, United States \\ bNaval Research Laboratory, Department of Defense, NRLSSC Code 7330, \\ Stennis Space Center, Mississippi 39529, United States
}

Harmful algal blooms (HAB) are defined as an increase in the concentration of a phytoplankton species in a water body that could be triggered by many environmental, meteorological, and hydrological factors simultaneously, such as eutrophication, sea surface temperature, and wind and current directions. HAB issues are global-appearing in locations such as Lake Tai in China, Lake Erie in the United States, and Lake Victoria in Africa - and seem to be increasing in severity and extent under the impact of global changes. These issues result in many unintended environmental, economic, ecological, and human health consequences. They include but are not limited to mass mortalities of fish and marine mammals; economic loss due to reduced tourism, fish stocks, and shellfish harvests; degraded drinking water quality; and a suite of public health problems associated with the consumption of contaminated fish, drinking water, and shellfish via direct/indirect exposure to toxins such as Mycrocystis. These HABs may alter marine/coastal benthic habitats and ecosystem health through shading effect, generate adverse effects on submerged and/or emergent aquatic vegetation, impact various life stages of fish, and cause induced anoxia via overgrowth. Near-surface HABs, like the Karenia spp. blooms, can be easily detected and tracked by space-borne and airborne remote sensing technologies. Yet subsurface HABs, like the Dinophysis spp. blooms, cannot be detected directly with space-borne remote sensing. Both types of HABs, however, can appear as sudden events without early warning and persist in the water body for a period of time.

Remote sensing technologies and numerical modeling methods are adding significantly to our ability to investigate coupled biogeophysical and biogeochemical processes. Given the complexity of the coupling processes, combing various remote sensor types such as multispectral, hyperspectral, infrared, light detection and ranging (LIDAR), and microwave technologies will be required to address HAB development and propagation. New advancements in autonomous buoys, unmanned aerial vehicles (UAV), and autonomous underwater vehicles (AUV) are providing higher spatial and temporal sampling that was previously only obtainable with manned aircraft. When augmented with numerical hydrodynamic models, the ability to track, monitor, and predict the movement of HABs is increasing significantly and will undoubtedly improve HAB early warning capacities. However, persistent or sporadic cloud cover can potentially cause data gaps in passive remotely sensed data. To improve the spatial, temporal, and spectral resolution, multisensor image fusion and data mining could become inevitable tools for a wealth of feature extraction and content-based mapping.

In line with these directions, the six papers in this special section deal with different technologies and research issues all aimed at improving our understanding, detection, and monitoring capability of HABs. To improve the chlorophyll-a retrieval algorithms for high solar zenith angle conditions, $\mathrm{Li}$ et al., examine the existing chlorophyll-a retrieval algorithms and demonstrates that there is a need to modify them with respect to high solar zenith angle conditions. A common problem associated to the application of such mapping technology for chlorophyll-a retrieval is the need to eliminate the effects of atmosphere. Thus, a remote sensed algal bloom monitoring system needs a simple algorithm which is nonsensitive to atmospheric correction applicable to small aquatic systems. Ogashawara et al. present a slope algorithm to map algal blooms in inland waters to resolve this issue. However, to compensate for the limitations

(C) 2017 Society of Photo-Optical Instrumentation Engineers (SPIE) 
of optical remote sensing when restricted by cloud cover, it is worthwhile to use synthetic aperture radar (SAR), which can penetrate clouds, for possible monitoring of cyanobacterial blooms. In the SAR study, which is the very first one in this topical area of importance, a satellite-ground synchronous experiment has been conducted in Lake Tai by Wang et al. They establish that the cyanobacterial scum effectively attenuates capillary waves and appear as dark patches in SAR images, and therefore SAR has the potential to be developed as a tool for the remote sensing of algal scum. From a HAB detection perspective, two more papers focus on the characterization of Karenia brevis blooms on the West Florida Shelf using ocean color satellite imagery. Multitemporal observations obtained with the Moderate Resolution Imaging Spectroradiometer (i.e., MODIS-Aqua), are used to delineate HABs by combining remote-sensing reflectance at $555 \mathrm{~nm}$ and normalized fluorescence line height by Soto et al., which is further modified by and $\mathrm{Hu}$ and Feng. Finally, Xu et al. presented an interesting study for the verification of MODISbased monitoring precision over a green tide event in the Yellow Sea with the aid of UAV showing a success of data merging for characterization of the plume movement.

All of these papers show the deepened understanding of the topic in this special section and are advancing the remote sensing of HABs in our lakes and coastal waters. Both contribution and dedication of authors and reviewers are here deeply acknowledged.

Ni-Bin Chang is a professor with the University of Central Florida, Orlando, Florida, United States. He has been the co-chair for the SPIE Conference on Remote Sensing and Modeling of Ecosystems for Sustainability since 2012. He was an invited speaker for the SPIE Photonic Innovations and Solutions for Complex Environments and Systems Symposium, SPIE Remote Sensing Systems Engineering Symposium, and SPIE Conference on Satellite Data Compression, Communications, Processing. His research areas include image restoration, image enhancement, image fusion, data assimilation, cross-mission data merging, integrated image fusion, and data mining with remote sensing data for various environmental, ecological, and hydrological monitoring applications. He is a Fellow of SPIE.

Alan Weidemann is an oceanographer with the Naval Research Laboratory at Stennis Space Center, Mississippi, and has worked in sensing of bio-optical properties and processes in lakes and the ocean for the past 30 years. His research has been in the development and application of technology to better understand and characterize properties influencing light propagation and remote sensing signals in various hydrologic environments. Current research involves the use of shipboard LIDAR to characterize subsurface particle layers and fronts and their associated bio-optical-physical interactions. 